\title{
Front Matter: Volume 9255
}

, "Front Matter: Volume 9255," Proc. SPIE 9255, XX International Symposium on High-Power Laser Systems and Applications 2014, 925501 (10 February 2015); doi: 10.1117/12.2185755

SDIE Event: XX International Symposium on High Power Laser Systems and SPIE. Applications, 2014, Chengdu, China 


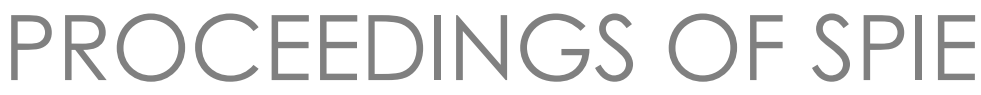

\title{
XX International Symposium on High-Power Laser Systems and Applications 2014
}

\author{
Chun Tang \\ Shu Chen \\ Xiaolin Tang \\ Editors
}

\section{5-29 August 2014 \\ Chengdu, China}

Organized by

China Academy of Engineering Physics (China)

The Chinese Optical Society (China)

Sponsored by

Key Laboratory of Science and Technology on High Energy Laser, China Academy of Engineering Physics (China) • Research Center of Laser Fusion, China Academy of Engineering Physics (China) • Dalian Institute of Chemical Physics, Chinese Academy of Sciences (China) • Institute of Optics and Electronics, Chinese Academy of Sciences (China) • Shanghai Institute of Optics and Fine Mechanics, Chinese Academy of Sciences (China) • Tsinghua University (China) • Sichuan University (China) • Institute of Fluid Physics, China Academy of Engineering Physics (China) • Beijing Institute of Applied Physics and Computational Mathematics (China) - Institute of Applied Electronics, China Academy of Engineering Physics (China)

Published by

SPIE

Volume 9255 
The papers included in this volume were part of the technical conference cited on the cover and title page. Papers were selected and subject to review by the editors and conference program committee. Some conference presentations may not be available for publication. The papers published in these proceedings reflect the work and thoughts of the authors and are published herein as submitted. The publisher is not responsible for the validity of the information or for any outcomes resulting from reliance thereon.

Please use the following format to cite material from this book:

Author(s), "Title of Paper," in XX International Symposium on High-Power Laser Systems and Applications 2014, edited by Chun Tang, Shu Chen, Xiaolin Tang, Proceedings of SPIE Vol. 9255 (SPIE, Bellingham, WA, 2015) Article CID Number.

ISSN: 0277-786X

ISBN: 9781628413229

Published by

SPIE

P.O. Box 10, Bellingham, Washington 98227-0010 USA

Telephone +1 3606763290 (Pacific Time) · Fax +1 3606471445

SPIE.org

Copyright (@ 2015, Society of Photo-Optical Instrumentation Engineers.

Copying of material in this book for internal or personal use, or for the internal or personal use of specific clients, beyond the fair use provisions granted by the U.S. Copyright Law is authorized by SPIE subject to payment of copying fees. The Transactional Reporting Service base fee for this volume is $\$ 18.00$ per article (or portion thereof), which should be paid directly to the Copyright Clearance Center (CCC), 222 Rosewood Drive, Danvers, MA 01923. Payment may also be made electronically through CCC Online at copyright.com. Other copying for republication, resale, advertising or promotion, or any form of systematic or multiple reproduction of any material in this book is prohibited except with permission in writing from the publisher. The CCC fee code is 0277-786X/15/\$18.00.

Printed in the United States of America.

Publication of record for individual papers is online in the SPIE Digital Library.

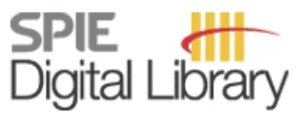

SPIEDigitallibrary.org

Paper Numbering: Proceedings of SPIE follow an e-First publication model, with papers published first online and then in print. Papers are published as they are submitted and meet publication criteria. A unique citation identifier (CID) number is assigned to each article at the time of the first publication. Utilization of CIDs allows articles to be fully citable as soon as they are published online, and connects the same identifier to all online, print, and electronic versions of the publication. SPIE uses a six-digit CID article numbering system in which:

- The first four digits correspond to the SPIE volume number.

- The last two digits indicate publication order within the volume using a Base 36 numbering

system employing both numerals and letters. These two-number sets start with 00, 01, 02, 03, 04, $05,06,07,08,09,0 A, 0 B \ldots$. OZ, followed by 10-1Z, 20-2Z, etc.

The CID Number appears on each page of the manuscript. The complete citation is used on the first page, and an abbreviated version on subsequent pages. 


\title{
Contents
}

\section{Part One}

\author{
xiii Authors \\ xix Conference Committee \\ xxi Introduction
}

SESSION 1 SOLID STATE LASER SOURCES AND SYSTEM ENGINEERING (INCLUDING DISK, FIBER, HYBRID LASERS)

925502 Theoretical and experimental study on reabsorption effect and temperature characteristic of a quasi-three-level 946nm Nd:YAG laser [9255-10]

925503 1540-nm single frequency single-mode pulsed all fiber laser for coherent Doppler lidar [9255-26]

925504 A linearly-polarized directly diode-pumped L-shaped Er:YAG laser at $1617 \mathrm{~nm}$ with average output power scaling [9255-62]

925505 Progress on the suppression of FM-to-AM modulations in SG-III laser facility [9255-110]

925506 High purity efficient first Stokes Raman laser [9255-123]

925507 High power mid-infrared continuous-wave optical parametric oscillator pumped by fiber lasers [9255-231]

$925508 \quad 1047 \mathrm{~nm} \mathrm{270mJ} \mathrm{all} \mathrm{solid} \mathrm{state} \mathrm{diode} \mathrm{pumped} \mathrm{MOPA} \mathrm{at} 50 \mathrm{~Hz}$ [9255-234]

925509 Passively Q-switched high-energy all-solid-state holographic Nd:YAG laser with a multiloop cavity [9255-253]

9255 OA 1 KHz high average power single-frequency Nd:YAG laser [9255-255]

$9255 \mathrm{OB} \quad 1.4 \mathrm{~kW}$ all-fiber narrow-linewidth polarization-maintained fiber amplifier [9255-262]

9255 0C Thermal management of liquid direct cooled split disk laser [9255-298]

9255 OD $\quad 160 \mathrm{~mJ}$ and 9ns electro-optics Q-switched conductively cooled $1047 \mathrm{~nm}$ Nd:YLF laser [9255-13]

9255 OE Precise tuning technique of frequency conversion crystals of SG facility [9255-21]

9255 OF Investigation on graded reflectivity mirror unstable laser resonators with larger intra-cavity phase aberrations [9255-46] 
9255 OG Development of large-aperture electro-optical switch for high power laser at CAEP [9255-89]

$9255 \mathrm{OH}$ Design and analysis on thermal adaptive clamping device for PPMgLN crystal used in solid state laser [9255-92]

92550 The study of the thermally expanded core technique in end-pumped $(\mathrm{N}+1) \times 1$ type combiner [9255-120]

9255 0J Novel front end design for synchronized output pulses with zero timing jitter in XG-III laser facility [9255-124]

9255 OK Research of high brightness 1018nm ytterbium doped fiber laser [9255-127]

$9255 \mathrm{OL} \quad$ Experimental design of double-cladding planar waveguide laser amplifier [9255-143]

9255 OM An improved method for stripping cladding light in high power fiber lasers [9255-149]

$9255 \mathrm{ON}$ Study on the thermal distribution and thermal management of high average power fiber lasers [9255-152]

$925500 \quad$ Home-made high efficiency side-pumped fiber amplifiers [9255-178]

9255 OP Numerical analysis of the convergence speed of the SPGD algorithm with two different perturbation methods in coherent beam combination using active segmented mirror [9255-192]

$92550 Q \quad$ Numerical analysis to four-wave mixing induced spectral broadening in high power fiber lasers [9255-213]

9255 OR Nd:YAG thin-disk laser with large dynamic range unstable resonance [9255-215]

9255 OS Experimental characterization of beam quality of a Yb:YAG thin disk laser [9255-226]

9255 OT Numerical approach to temperature and thermal stress in direct-liquid-cooled Nd:YAG thin disk laser medium [9255-241]

$9255 \mathrm{OU}$ All-fiber wavelength-tunable Tm/Ho-codoped laser between $1727 \mathrm{~nm}$ and $2030 \mathrm{~nm}$ [9255-266]

SESSION 2 HIGH POWER DIODE LASER

9255 OV HiLASE: development of fully diode pumped disk lasers with high average power (Invited Paper) [9255-17]

9255 OW A 3000W 808nm QCW G-stack semiconductor laser array [9255-223]

$92550 X \quad$ Packaging of complete indium-free high reliable and high power diode laser array [9255-309]

9255 OY Research on 9xx nm diode laser for direct and pumping applications [9255-311] 
925510 Generation of 6.05J nanosecond pulses at a $1 \mathrm{~Hz}$ repetition rate from a cryogenic cooled diode-pumped Yb:YAG MOPA system [9255-292]

925511 Progress on the XG-III high-intensity laser facility with three synchronized beams (Invited Paper) [9255-82]

925512 Production of petawatt laser pulses by backward Raman amplification in plasma [9255-27]

925513 Generation of high average power supercontinuum involve visible spectrum [9255-170]

925514 Spectral shaping of the amplified signal in optical parametric chirped pulse amplification [9255-227]

925515 Application problems of object-image-grating self-tiling in ultra-short pulse lasers [9255-304]

$925516 \quad$ Method to realize real-time monitoring and control of a tiled-grating compressor for the XGIII laser facility 9255-45]

925517 Improvement of temporal contrast by 2 order of magnitude based on the deformable mirrors [9255-80]

925518 Array element auto-tiling based on capacitive displacement sensor [9255-122]

925519 Amplitude instability of the dissipative soliton mode-locked fiber laser [9255-133]

$92551 \mathrm{~A}$ Effect of acoustic waveguide properties on the Brillouin gain spectrum in multi-mode fibers [9255-66]

9255 1B Eigenmodes of large-mode-field laser resonators with intra-cavity phase aberrations [9255-72]

9255 1C Theoretical study of mode evolution properties in a $3 \times 1$ adiabatic tapered single-mode fiber combiner [9255-279]

9255 1D Analysis of thermal stresses in $\mathrm{HfO}_{2} / \mathrm{SiO}_{2}$ high reflective optical coatings for high power laser applications [9255-3]

$92551 \mathrm{E}$ Investigation of defect rate of lap laser welding of stainless steel railway vehicles car body [9255-6]

9255 IF Research on power spectral density after nonlinear propagation in high-power solid state laser [9255-16]

$92551 \mathrm{G}$ Laser superposition in multi-pass amplification process [9255-18]

$92551 \mathrm{H} \quad$ Simulation code of high power discharge for iPhones [9255-69]

$925511 \quad$ High precision pointing system based on Risley prism: analysis and simulation [9255-119] 
$92551 \mathrm{~J} \quad$ Numerical analysis of the beam quality and spectrum of wavelength-beam-combined laser diode arrays [9255-130]

$92551 \mathrm{~K}$ Theoretical study of iterative pump number of diode pumped solid state laser [9255-159]

$9255 \mathrm{lL}$ Compensation analysis of low frequency distorted wave front of laser beam [9255-174]

$92551 \mathrm{M}$ Research of beam control system component simulation and separation method of the kinematics coupling [9255-185]

9255 IN Research on 3D simulation of coupling model among flow field, optical field and heating effects of mirrors [9255-198]

925510 Theoretical analysis and comparison of Tm-doped fiber lasers with different pump bands [9255-244]

$9255 \mathrm{lP}$ Influence of the neodymium glass parameters on the amplified spontaneous emission in slab amplifier [9255-281]

$92551 Q$ Mathematical simulation of heating effects in a static diode-pumped vapor rubidium cell [9255-300]

9255 IR Efficient fourth harmonic generation of Nd: glass lasers in ADP and DKDP crystals [9255-182]

SESSION 4 GAS AND CHEMICAL LASERS (INCLUDING DPALS)

9255 is DPAL activities in Japan (Invited Paper) [9255-49]

9255 IT Molecular singlet delta oxygen quenching kinetics in the EOIL system [9255-34]

$92551 \mathrm{U} \quad$ Precision beam pointing control with jitter attenuation by optical deflector exhibiting dynamic hysteresis in COIL [9255-61]

$92551 \mathrm{~V} \quad \mathrm{O}_{2}\left({ }^{1} \Delta_{g}\right)$ detection using broadband CARS [9255-113]

9255 IW Study on key operating parameters of diode-pumped Cs vapor laser [9255-142]

9255 1X DPAL research in Changsha [9255-230]

$92551 Y$ Continuously wavelength tunable high pressure $\mathrm{CO}_{2}$ lasers [9255-237]

$925512 \quad$ Features of power extraction in EOIL [9255-251]

925520 Semi-analytical and CFD model calculations of subsonic flowing-gas DPALs and their comparison to experimental results [9255-256]

$925521 \quad$ Hydrogen fluoride chemical laser cascade dynamics under cavity loss modulation [9255-257]

925522 The research on the technology of surface discharge optical pumping source [9255-268] 
925523 Progress on high power excimer laser in NINT [9255-275]

925524 Theoretical investigation of diffraction features of a planar wave using a Reflecting Volume-Bragg-Grating (RVBG) [9255-299]

925525 A quasi-CW linearly polarized rubidium vapor laser pumped by a 5-bar laser diode stack [9255-50]

925526 Research on COIL employing no-flake-nozzle and $\mathrm{CO}_{2}$ as buffer gas [9255-54]

925527 Chemical oxygen-iodine laser with a cryosorption vacuum pump with different buffer gases [9255-63]

925528 Modeling of lasing possibility in XeF(C-A) amplifier of the THL-100 laser system [9255-65]

925529 Small signal gain measurement of liquid oxygen under different wavelength laser pump [9255-90]

9255 2A Single line oscillation of continuous wave HF chemical laser with a grating cavity [9255-103]

9255 2B Data acquisition and control system with a programmable logic controller (PLC) for a pulsed chemical oxygen-iodine laser [9255-104]

9255 2C Optical cavity temperature measurement based on the first overtones spontaneous emission spectra for HF chemical laser [9255-108]

9255 2D Optical resonator with nonuniform magnification for improving beam uniformity of chemical oxygen iodine lasers [9255-109]

$92552 \mathrm{E}$ Spectral analysis of cavity chemiluminescence of a combustion-driven HF laser fueled by $\mathrm{NF}_{3}[9255-111]$

$92552 \mathrm{~F} \quad$ Oxygen assisted iodine atoms production in an RF discharge for a cw oxygen-iodine laser [9255-132]

$92552 \mathrm{G}$ The amplification of stimulated Raman scattering in $\mathrm{H}_{2}$ pumped by a Q-switched Nd:YAG laser [9255-135]

$92552 \mathrm{H} \quad$ Blue satellites of absorption spectrum study of sodium based excimer-pumped alkali vapor laser [9255-139]

$925521 \quad \mathbf{H}_{2}$ stimulated Raman scattering in a multi-pass cell [9255-144]

$92552 \mathrm{~J}$ lodine flow rate measurement for COIL with the chemical iodine generator based on absorption spectroscopy [9255-146] 


\section{Part Two}

9255 2K Investigation on the separation performance of strongly swirling flow singlet oxygen generator [9255-150]

9255 2L Investigation on regeneration of basic hydrogen peroxide by electrochemical methods [9255-151]

$92552 \mathrm{M}$ Research on reaction zone structure of high-press gravity-independent singlet oxygen generator [9255-153]

$92552 \mathrm{~N} \quad$ Experimental performances of pre-swirling jet singlet oxygen generator [9255-154]

925520 Multi-photon processes in alkali metal vapors [9255-157]

$92552 \mathrm{P}$ Feasibility study of a novel pressure recovery system for $\mathrm{CO}_{2}$-COIL based on chemical absorption [9255-158]

$92552 \mathrm{Q}$ Measuring laser beam quality by use of phase retrieval and Fraunhofer diffraction [9255-161]

$92552 R \quad 420 \mathrm{~nm}$ alkali blue laser based on two-photon absorption [9255-162]

925525 Tuning laser output characteristics of a pyrotechnically pumped free-running Nd:YAG laser in terms of pumping kinetics [9255-179]

$92552 T$ The mode-matching model of diode-end-pumped alkali vapor lasers [9255-199]

$92552 \mathrm{U} \quad$ High repetition ration solid state switched $\mathrm{CO}_{2}$ TEA laser employed in industrial ultrasonic testing of aircraft parts [9255-238]

$92552 \mathrm{~V}$ Efficient gas lasers pumped by run-away electron preionized diffuse discharge [9255-250]

$92552 \mathrm{~W}$ Tunable diode-laser spectroscopy (TDLS) of $811.5 \mathrm{~nm}$ Ar line for $\operatorname{Ar}\left(4 \mathrm{~s}[3 / 2]_{2}\right)$ number density measurements in a 40MHz RF discharge [9255-259]

9255 2X Discharge initiated high power repetitively pulsed HF/DF laser [9255-263]

\section{SESSION 5 RESONATORS AND LASER BEAM CONTROL}

$92552 Y \quad B e a m$ quality active control of a slab MOPA solid state laser [9255-189]

$92552 Z$ Thermal blooming effects of gas on laser propagation in a closed tube [9255-74]

925530 A new method of frequency-doubling with converging cavity [9255-201]

925531 Thermal lens effect induced by high power diode laser beam in liquid ethanol and its influence on a probe laser beam quality [9255-217] 
925532 Influence of distribution of optical component surface defects on near field beam quality [9255-295]

925533 Simulation research on beam steering technology based on optical phased array [9255-78]

925534 The application of ptychography in the field of high power laser [9255-267]

925535 Dependence of the influence function on laser-induced thermoelastic deformation of deformable mirrors [9255-282]

925536 Analysis of atmospheric turbulence anisoplanatism [9255-83]

925537 Phase-distortion correction based on stochastic parallel proportional-integral-derivative algorithm for high-resolution adaptive optics [9255-112]

925538 Double deformable mirrors' control based on voltage decoupling for adaptive optics system [9255-163]

925539 A fast high voltage driver for the piezoelectric fast steering mirror [9255-167]

9255 3A Investigation of anisoplanatic effect in adaptive optics for atmospheric turbulence correction [9255-168]

9255 3B Wavefront reconstruction with total-least square method in an adaptive optic system with HS wavefront sensor [9255-187]

$92553 \mathrm{C}$ A transformation approach for aberration-mode coefficients of Walsh functions and Zernike polynomials [9255-193]

9255 3D Experimental study on return signals detection of pulsed sodium laser beacon [9255-200]

$92553 \mathrm{E}$ The entire beam wavefront control of high power laser facility [9255-207]

$92553 \mathrm{~F} \quad$ Unload control of double fast steering mirrors of the control system [9255-35]

$92553 \mathrm{G}$ Simulations of far-field optical beam quality influenced by the thermal distortion of the secondary mirror for high-power laser system [9255-202]

$92553 \mathrm{H} \quad$ Discriminatively trained part based model armed with biased saliency [9255-44]

$925531 \quad$ Automatic alignment technology in high power laser system [9255-85]

9255 3J High-accuracy arithmetic for cavity mirror automatic alignment in multi-pass beam system [9255-86]

9255 3K A novel multi-view object recognition in complex background [9255-115]

$92553 \mathrm{~L}$ Simulation of phase noise for coherent beam combination [9255-121]

$92553 \mathrm{M}$ Simulation study on fiber-coupling efficiency for quantum communication through atmospheric turbulence [9255-128] 
$92553 \mathrm{~N}$ Investigation on the influence of spectral linewidth broadening on beam quality in spectral beam combination [9255-195]

925530 Characteristic analysis of a polarization output coupling Porro prism resonator [9255-15]

$92553 \mathrm{P} \quad$ Vibration characteristic of high power $\mathrm{CO}_{2}$ laser [9255-148]

SESSION 6 APPLICATIONS, LASER MATERIALS (CRYSTAL, CERAMIC), NOVEL APPROACHES

$92553 Q \quad$ Laser space debris removal: now, not later (Invited Paper) [9255-500]

$92553 R \quad$ Mean flow aero-optic effect of airborne optical plafform [9255-60]

925535 Peculiarities of radiation formation in laser systems for remote sensing [9255-145]

9255 3T Laser assisted die bending: a new application of high power diode lasers (Invited Paper) [9255-273]

$92553 \mathrm{U}$ Interaction of extreme ultraviolet laser radiation with solid surface: ablation, desorption, nanostructuring (Invited Paper) [9255-285]

9255 3V Photoluminescence defects on subsurface layer of fused silica and its effects on laser damage performance [9255-233]

9255 3W Crystalline micro/nanostructures fabrication on silicon using femtosecond laser [9255-294]

$92553 \times$ Regular sub-wavelength surface structures induced by femtosecond laser pulses on nickel [9255-11]

$92553 Y$ Study of high power laser mirror shape maintenance technology [9255-173]

925532 Microstructure and properties of welds between $\mathbf{5 7 5 4}$ Al alloys and AZ31 Mg alloys using a Yb:YAG laser [9255-261]

925540 Femtosecond laser induced surface structuring on silicon by diffraction-assisted micropatterning [9255-297]

$925541 \quad$ A novel description based on skeleton and contour for shape matching [9255-79]

925542 Mechanical analysis of photo-electricity measure equipment shafting in mobile-platform [9255-94]

925543 Realization of LOS (Line of Sight) stabilization based on reflector using carrier attitude compensation method [9255-129]

925544 PCIE interface design for high-speed image storage system based on SSD [9255-156]

925545 Investigation into the initiation of hexanitrostilbene by laser-driven composite flyer plates [9255-211] 
925546 The structure design and performance analysis for damping system of the airborne equipment [9255-125]

925547 Study about the 6-DOF parallel tracking platform [9255-137]

925548 Laser erosion diagnostics of plasma facing materials with displacement sensors and their application to safeguard monitors to protect nuclear fusion chambers [9255-93]

925549 Interaction of high-power ultrashort laser pulses with air [9255-312]

9255 4B Research of beam smoothing technologies using CPP, SSD, and PS [9255-42]

9255 4C Research on auto-focusing of high signal to noise ratio image with weak light [9255-55]

9255 4D A multi-beam alignment method at target area for ICF laser facility based on invariant subarea configuration of the alignment sensor [9255-91]

$92554 \mathrm{E} \quad$ A target positioning method for ICF laser facility without translational kinematic coupling [9255-126]

$92554 \mathrm{~F}$ Experimental research on high-contrast measurement for ns high-power laser shaped pulse [9255-136]

9255 4G Lifetime of high-power GaAs photoconductive semiconductor switch triggered by laser of different power density [9255-175]

$92554 \mathrm{H}$ Optical transmission and reflection of a plasma produced in nanosecond laser induced air breakdown [9255-219]

92554 Three-dimensional footprint of optical breakdown in transparent dielectrics induced by femtosecond pulsed lasers and the effect of laser energy absorption [9255-224]

$92554 \mathrm{~J}$ Research on high-power laser diode used for triggering photoconductive semiconductor switch [9255-97]

9255 4K Energy transmission by laser [9255-269]

$92554 \mathrm{~L}$ Temperature dependence of Raman scattering in KDP crystal [9255-118]

9255 4M Measurement of crystal defects using phase retrieval technique [9255-280]

9255 4N Design and simulations of CAEP THz FEL resonator [9255-39]

$925540 \quad$ High-peak-power terahertz sources pumped by high power laser and single-shot measurement of terahertz temporal waveform [9255-190]

$92554 \mathrm{P}$ Aspects for efficient wide spectral band $\mathrm{THz}$ generation via $\mathrm{CO}_{2}$ laser down conversion [9255-140]

$92554 Q$ Study on conversion efficiency of optical-to-terahertz in optical rectification [9255-291] 
$92554 R$ 3D coordinate transform model of optical images fusing vector distance information [9255-47]

92554 Radiative characteristics of high-power halogen tungsten lamp used in calibration of highenergy laser energy meter [9255-68] 


\section{Authors}

Numbers in the index correspond to the last two digits of the six-digit citation identifier (CID) article numbering system used in Proceedings of SPIE. The first four digits reflect the volume number. Base 36 numbering is employed for the last two digits and indicates the order of articles within the volume. Numbers start with 00, 01, 02, 03, 04, 05, 06, 07, 08, 09, OA, OB...0Z, followed by 10-1Z, 20-2Z, etc.

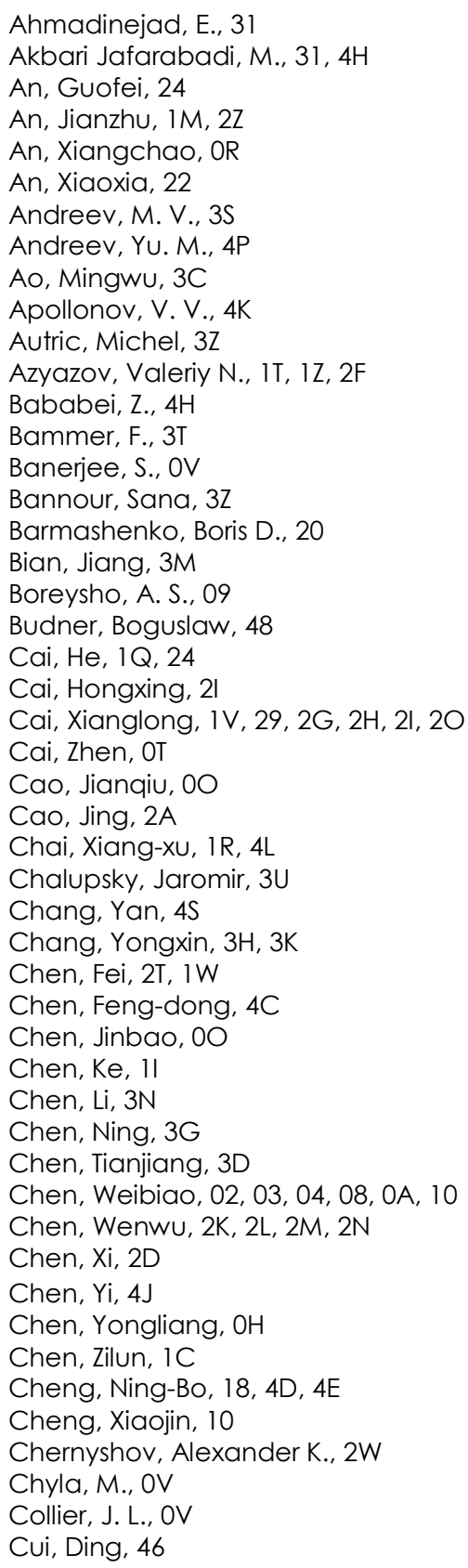

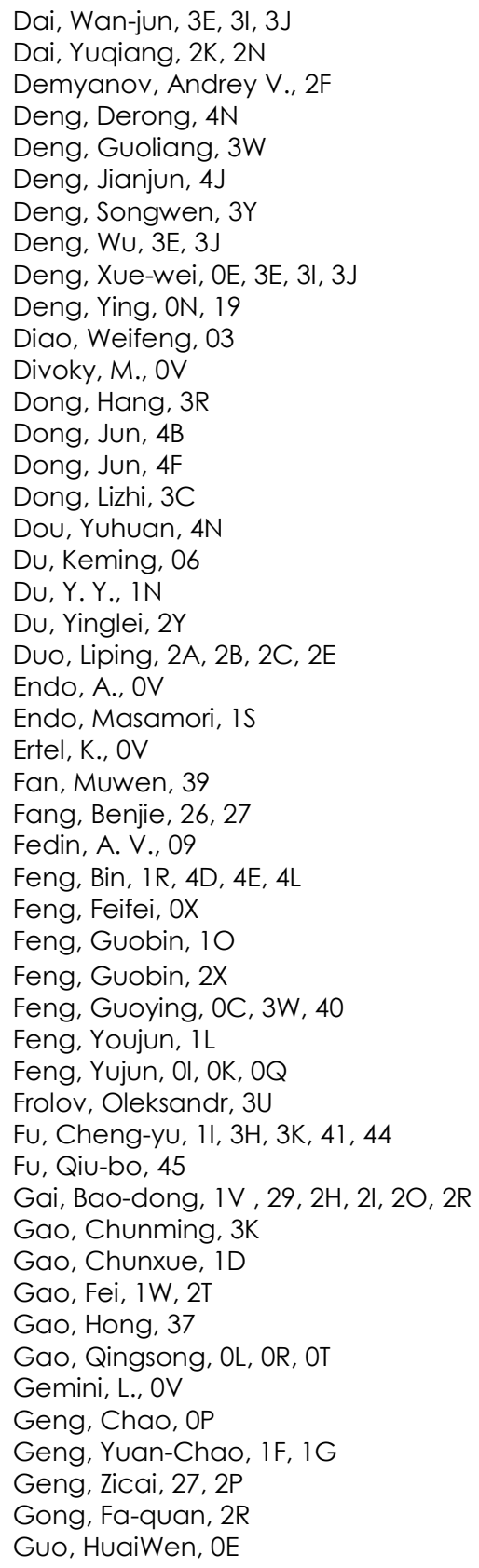

\section{Downloaded From: https://www.spiedigitallibrary.org/conference-proceedings-of-spie on 25 Apr 2023
Terms of Use: https://www.spiedigitallibrary.org/terms-of-use


Guo, Jing, 1W

Guo, Jing-wei, 1K, 1V, 29, 2G, 2H, 2l,

$2 \mathrm{M}, 2 \mathrm{O}, 2 \mathrm{R}, 30$

Guo, Ruhai, 3G

Guo, Shaofeng, 00

Guo, Yi, 0J, 11

Han, Juhong, 1Q, 24

Han, Wei, IR

Hatsugai, Takuro, $1 \mathrm{H}$

$\mathrm{He}, \mathrm{Bi}, 45$

He, Xin, IU, 2Q

$\mathrm{He}$, Yang, $2 \mathrm{~T}$

$\mathrm{He}$, Zhongwu, $2 Y$

Heaven, M. C., $1 T$

Hernandez-Gomez, C., OV

Hou, Dong, OW, OX

Hou, Jing, OU, $1 C$

Hou, Xia, 03, 04

$\mathrm{Hu}$, Dapeng, $2 \mathrm{~K}$

$\mathrm{Hu}$, Dongxia, 05, 3E, 3J, 4B

$\mathrm{Hu}, \mathrm{HaO}, \mathrm{OL}$

Hu, Hongyi, 42

Hu, Jinlong, 41

Hu, Peng, $2 Z$

Hu, Qi-qi, 3L

$\mathrm{Hu}$, Shijie, 3A

$\mathrm{Hu}$, Shu, 29, 2G, 2H, 2l, 2O, 2R

$\mathrm{Hu}$, Xiao Yang, $4 \mathrm{~S}$

Hu, Yun, 23

Hua, Weihong, $1 \mathrm{X}$

Huai, Ying, 2D

Huang, Chao, 22, 23, $2 X$

Huang, Dequan, 3D

Huang, Jin, 3V

Huang, Jing, 02

Huang, $\mathrm{Ke}, 22,2 \mathrm{X}$

Huang, Kui, 3A

Huang, Linhai, 39

Huang, Wan-Qing, 1F, $1 G$

Huang, Wei, 25

Huang, Wenfa, 10

Huang, Xiaojun, 0J, 11

Huang, Zheng, 0J, 11

Huang, Zhihe, 00

Huang, Zhihua, 13, 19

Huang, Zhi-meng, 3L

Huang, Zili, 4R

Hülsewede, R., OY

Huo, Yihua, 4R

Huynh, J., OV

Ivanov, N. G., 28

lyoda, Mitsuhiro, $1 \mathrm{H}$

Jambunathan, $\mathrm{V}_{\text {., }} \mathrm{OV}$

Jia, Chun-yan, 2R

Jia, Huai-Ting, OE, 1R, 4D, 4E

Jia, Shuqin, 2D

Jiang, Dongbin, 0J, 11

Jiang, Ming, 45

Jiang, Weiwei, 42

Jiang, Xiaodong, 3V
Jiang, Xuejun, 0J, 11

Jiang, Zongfu, 35

Jiao, Zhihong, 12

Jin, YU-Qi, 1U, 1V, 26, 27, 29, 2A, 2B, 2C, 2D, 2G,

$2 \mathrm{H}, 2 \mathrm{I}, 2 \mathrm{~J}, 2 \mathrm{~K}, 2 \mathrm{~L}, 2 \mathrm{M}, 2 \mathrm{~N}, 2 \mathrm{O}, 2 \mathrm{P}, 2 \mathrm{Q}, 30,37,3 \mathrm{Y}$

Jing, Feng, 11, 17, 18, 3E

Juha, Libor, 3U

Kang, Xiaoli, $2 S$

Kang, Yuanfu, 2B

Kasuya, Koichi, 48

Kazemi, Sh., OS

Ke, Changchun, 2K, 2L, 2M

Ke, Wei-Wei, OK, OQ, 1A, $1 \mathrm{~J}$

Kochetov, Igor V., 2F

Kolacek, Karel, $3 \mathrm{U}$

Korczyc, Barbara, 48

Lanskii, G. V., 4P

Lebedev, V. F., 09

Leng, Jinyong, 00

Li, Chao, ON, 13, 19

Li, Daijun, 06

Li, Dian-jun, IW, 2T

Li, Fu-Quan, 1R, 4D, 4E, 4L

Li, Gang, 3Y

Li, Guofu, 2B, 2E

Li, Hui, 29, 2G, , 2H, 2l, $2 \mathrm{O}$

$\mathrm{Li}$, Jianmin, $3 \mathrm{~N}$

Li, Jin-ying, 11

Li, Lei, 10

Li, Lei, 21

Li, Liucheng, 2B, 2C, 2E

Li, M. Z., 05

Li, Mei, 39

Li, Ming, 4N

Li, Ping, 4B

Li, Qing, 0J, 11

Li, Qingwei, 2P

Li, S. X., IN

Li, Shiguang, OA

Li, Shuang, 1D

$\mathrm{Li}$, Tenglong, OI, OK, OM, OQ

Li, Xiao, 07

Li, Xiaoning, OX

Li, Xinyang, OP, 38, 39, 3A

Li, Y., $1 \mathrm{~N}$

Li, Yi-min, $2 R$

Li, Yongzhao, 27, 2P

Li, Ze-Ren, $4 \mathrm{O}$

Li, Zhaoyang, 15

Li, Zhilin, 16, 18

Li, Zhiyong, 25

Lian, Bo, 31

Liang, Xiaobao, ON, 13, 19

Liberatore, Ch., OV

Lin, Donghui, OG

Liv, Can-li, 4A

Liv, Cheng, 34

Liu, Chong, OT

Liu, Dong, 29, 21, 30

Liu, Guo-dong, 4C

xiv

Proc. of SPIE Vol. $9255925501-14$ 
Liv, Hongjie, 3V

Liu, Hongzhong, 16

Liv, Hua, 4F

Liu, Hui, OW

Liu, Jin, 10

Liu, Jinbo, 1V, 29, 2H, 2 I

Liu, Jingru, 22, 23, 2X

Liv, Jiqiao, 03

Liu, Lan-Qin, 12, 1F, $1 \mathrm{G}$

Liu, Lei, 07

Liu, Qiao, 40

Liu, Qin, 1U, 2Q

Liu, Qinyong, 06

Liu, Ruzhen, 42

Liu, Shunfu, $3 Y$

Liu, Tong, 2R

Liu, Wan-fa, 1K, 2H, 2O, 2R

Liu, Wenguang, 35

Liu, Xiaomeng, 06

Liu, Xingsheng, OW, OX

Liu, Yalong, $\mathrm{OX}$

Liu, Yi, 4G, 4J

Liu, Yuan, 03

Liu, Yushi, 2K, 2L, 2M, 2N, 3Y

Liu, Zejin, OB

Liu, Zhendong, 2K, 2M, 2N

Lomaev, Mikhail I., $2 \mathrm{~V}$

Long, Xuejun, 3M

Losev, V. F., 28, 3S, 4P

LU, Fei, 3N

Lu, Pei, 3H

Lu, Tingting, 08, OD

Lu, Yanhua, 3D

Lu, Zhong-gui, 4F

Lubenko, D. M., 4P

Lucianetti, A., OV

Luo, Jia, 3N

LUo, Jiangshan, $2 S$

Luo, Wen, OP

Luo, Xi, 39, 3A

Luo, Yong-quan, 3L

Luo, Zhongxiang, $2 Y$

LV, Guosheng, 2K, 2N

Lv, Jia-kun, 4F

Ma, Haotong, 3M

Ma, Jia-guang, 43

Ma, Jian, 08, OD

Ma, Lianying, 22, 23, $2 X$

Ma, Pengfei, OB

Ma, She, 46

Ma, Xiuhua, 08, OA, OD

Ma, Yan-Hua, 1U, 2Q

$\mathrm{Ma}, \mathrm{Yi}, \mathrm{Ol}, \mathrm{OK}, \mathrm{OM}, \mathrm{OQ}$

Mahdieh, M. H., OS, 31, 4H, 4I

Mao, Jian-Qin, $1 \mathrm{U}$

Mao, Yao, 43

Mason, P. D., OV

Masse, Jean-Eric, $3 z$

Mattei, Simone, $3 z$

Matvienko, Gennadii G., 49
Meng, Kun, 40

Meng, Qingkun, 1V

Meng, Qinglong, 4Q

Meusel, J., OY

Mezhenin, Andrey $\mathrm{V}$., 1T, $1 \mathrm{Z}$

Mhiri, Hatem, $3 Z$

Mi, Changwen, 1D

Mikheyev, Pavel A., 1T, 2F, 2W

Miura, T., OV

Mocek, T., OV

Morkel, Francois, 1Y, $2 \mathrm{U}$

Mortezaei, Z., 4I

Motokoshi, Shinji, 48

Mroz, Waldemar, 48

Mu, Jie, 16, 18

Nagisetty, S. S., OV

Nakai, Mitsuo, 48

Napartovich, Anatoly P., 2F

Nie, Zhiqiang, OW

Novak, O., OV

Pan, Xingchen, 34

Pan, Xudong, 33

Pan, Zhiyong, 00

Panchenko, Alexei N., 2V

Panchenko, Nikolai A., 2V

Panchenko, YU. N., 28, 3S, 4P

Peng, Qi, 11

Peng, Qi-Xian, 40

Peng, Xianrong, 41

Peng, Zhi-tao, 4C, 4F

Pershin, A. A., $1 T$

Phillips, P. J., OV

Phipps, Claude R., 3Q

Pietrzak, A., OY

Pilař, J., OV

Pogoda, A. P., 09

Puchikin, A. V., 35

Qi, Bo, 3M

Qi, Litao, 3X

Qian, Hang, 22, 23

Qin, Wen-Zhi, 45

Ran, Huanhuan, 4R

Rao, Changhui, 39

Ren, Ge, 3F, 3M, 47

Rosenwaks, Salman, 20

Rostohar, D., OV

Sang, Fengting, 1K, 26, 27, 2B, 2C, 2H, 2J, 2K, 2L,

$2 \mathrm{M}, 2 \mathrm{~N}, 2 \mathrm{O}, 2 \mathrm{P}$

Sato, Shunichi, $1 \mathrm{H}$

Sawicka, M., OV

Schmidt, Jiri, 3U

Schröder, M., OY

Schumi, T., 3T

Schuöcker, G., 3T

Schuöcker, D., 3T

Sebastian, J., OY

Severova, P., OV

Sha, Zi Jie, 4S

Shang, Jianli, OR, OT

Shang, Yaping, 07 
Shao, Bibo, 23

Shao, Li, 3A

Shen, Feng, 38

Shen, Yanlong, 22

Shen, Yi, 4G

Shi, Jingshui, 4G, 4J

Shi, Wen-Bo, 1U, 2Q

Shi, Xiangchun, 10

Shi, Zhe, 29, 2G, 2H, 2I, 20

Shu, Xiaojian, $4 \mathrm{~N}$

Sikocinski, P., OV

Slezak, O., OV

Smetanin, S. N., 09

Smrz, M., OV

Sperrer, G., 3T

Spitzer, O., 3T

Stehmann, Timo, $2 \mathrm{U}$

Straus, Jaroslav, 3U

Su, Hua, OF, IB

Su, Jingqin, $0 J, 11,12,16,17,18,4 B$

Sugimoto, Takuya, $1 \mathrm{H}$

Sun, Li Qun, 4S

Sun, Li, OJ, 11

Sun, Long, 3Y

Sun, Tianxiang, $3 Y$

Sun, Yang, 2D, 37

Sun, Yinghong, OK, OM

Sun, Yinhong, Ol, OQ

Sun, Zhi-hong, 4F

Suslov, Alexey I., 2V

Svabek, R., OV

Tan, Rongqing, 25

Tan, Sen, 47

Tan, Y., 3F

Tan, Yan-nan, 2H, 2O, 2R

Tan, Yi, OP, 42, 47

Tan, Yong, 21

Tang, Chun, OR, OT, IB

Tang, Jun, 4C, 4F

Tang, Shukai, 2A, 2B, 2C, 2E

Tang, T., 3F

Tang, Xuan, 1 A, 1 J, 3L

Tang, Ying, 22, $2 X$

Tang, Yongjian, $2 S$

Taniguchi, Seiji, 48

Taniwaki, Manabu, $1 \mathrm{H}$

Tao, Mengmeng, 10

Tao, Rumao, OB

Tarasenko, Victor F., $2 \mathrm{~V}$

Tian, Fei, 3N

Tian, Jing, 43

Tian, Junlin, 33

Tian, Xiaocheng, 05, 4B

Tian, Xiaogiang, 3D

Tokunaga, Kazutoshi, 48

Torbin, A. P., $1 T$

Tu, BO, OT

Ufimtsev, Nikolay I., 2F, 2W

Vanda, J., OV

von Bergmann, Hubertus, $1 Y, 2 U$
Vorontsova, Ekaterina A., 2W

Waichman, Karol, 20

Wan, Yuan, 02

Wang, Bing, $3 G$

Wang, Bingyan, $1 \mathrm{P}$

Wang, Bo-peng, 17

Wang, Chunrui, 2T

Wang, Dahui, 23

Wang, De-en, 17, 3E, 3J

Wang, Fang, $1 \mathrm{R}$

Wang, Fei, 10

Wang, Feng, 3Y

Wang, Fengrui, $3 \mathrm{~V}$

Wang, Haiyan, 34

Wang, Hongxiao, $1 \mathrm{E}$

Wang, Hongyan, 1X, 21

Wang, Hongyuan, $1 Q, 24$

Wang, J. J., 05

Wang, Jian, 2A, 2B, 2E

Wang, Jiangfeng, 10

Wang, Jianjun, 13

Wang, Jianlei, 10

Wang, Jinglong, 2K, 2L, 2M, 2N

Wang, Jingwei, OW, OX

Wang, Juntao, OL

Wang, Ke, OT

Wang, Li, IP

Wang, Li-quan, $1 R$

Wang, Meng, 45

Wang, Mingjian, 04

Wang, Sanhong, 3M

Wang, Shenglai, 4L

Wang, Shiming, 44

Wang, Shiwei, ON, 13, 19

Wang, Shuai, 3C

Wang, Shutong, 40

Wang, Wei, 4G, 4J

Wang, Wei-ping, 4A

Wang, Wen-feng, 4C

Wang, Wen-Yi, IF, $1 G$

Wang, Xiao, 0J, 11, 16, 18

Wang, Xiao-dong, 0J, 11, 17

Wang, Xiao-Jun, OF, OL, OQ, OT, 1A, 1B, 1J

Wang, Xiaolin, $\mathrm{OB}$

Wang, Yafei, 3H

Wang, Yanshan, OI, OK, OM, OQ

Wang, Yao, 45

Wang, You, 1Q, 24

Wang, Yuan-cheng, OE, 3E, 3I, 3J, 4B

Wang, Yuanhu, 2A, 2B, 2C, 2E

Wang, Zhenbao, 10

Wang, Zhenfu, OW

Wani, Fumio, 15

Wei, Binbin, 35

Wei, Fu-peng, 4C

Wei, Ji Feng, $4 S$

Wei, Xiao-Feng, ON, 12, 4D, 4E

Wei, Yu-Xin, OF, 1B

Wen, Jing, 0J, 11, 14, 17

Wittschirk, T., OY 
Wölz, M., OY

Wu, Chun-xia, 46

Wu, Dengsheng, OG

Wu, Jing, 2Y, 3B

Wu, Juan, OI, OK, OM, OQ

Wu, Ke-nan, 2D, 37

Wu, Weidong, $3 \mathrm{~V}$

Wu, Zhaohui, 12, 16

Xia, Liansheng, 4G, 4J

Xia, Yan-wen, $4 \mathrm{~F}$

Xiang, Rujian, 2Y, 3B

Xiang, Yong, 1R, 4D, 4E

Xie, Ji-jiang, IW, 2T

Xie, Kun, 35

Xie, Na, OJ

Xie, $\mathrm{Na}, 11,17$

Xie, Xiaogang, $1 \mathrm{M}$

Xie, Xingquan, 3J

Xie, Xudong, 11

Xiong, Lingling, OW, OW

$X \cup$, Bing, 3C

$X \cup$, Dangpeng, 05, 4B

$X U$, Honglai, $2 Y$

$X U$, Ming, $3 R$

$X U$, Mingxiu, 26, 27, $2 \mathrm{~J}$

$X U, X i a o b o, 2 K, 2 L, 2 M, 2 N$

$\mathrm{XU}, \mathrm{Xiaojun}, 07,1 \mathrm{X}$

$X U$, Xinguang, $4 \mathrm{~L}$

$X U, Y a n, 1 W, 2 T$

$\mathrm{XU}$, Zhiyong, 3H, 3K, 44

Xue, Guanghui, OU

Xue, Liangping, 1Q, 24

Xue, Quanxi, 23

Yan, Cong-lin, $\mathrm{OH}, 46$

Yan, Hong, 3N

Yan, Wei, 16

Yang, Fan, $2 S$

Yang, Guilong, 2T

Yang, Huomu, OC

Yang, Pengling, 10

Yang, Ping, 3C

Yang, Qi, 08, OD

Yang, Weiqiang, OU

Yang, Xianheng, 3W

Yang, Xingfan, 4N

Yang, Ying, 3E, 3I

Yang, Zhongguo, 10

Yang, Zining, $1 \mathrm{X}$

Yao, Yudong, 4M

Yastremskii, A. G., 28

Ye, Hongwei, 38

Ye, Rong, 4Q

Ye, Xisheng, 10, 22, 2X

Ye, Yidong, 3N

Ye, Zhibin, OT

Yi, Aiping, 22, 23, 2X

Yin, Ke, OU

You, Kewei, 32

Yu, Haijun, 2A, 2B, 2C, 2E

Yu, Hongliang, $2 B$
Yu, Huahua, $2 Z$

YU, Huapeng, 3H, 3K

YU, Li, 22, 23

YU, Yi, OR, OT

Yu, Zhenzhen, 04

Yuan, Hao-yu, 4B, 4C, 4F

Yuan, Qiang, 3E, 3I, 3J, 4B

Yue, Yufang, $1 \mathrm{M}$

Yun, Yu, 3D

Zemlyanov, Alexander A., 49

Zeng, Bing, $4 \mathrm{Q}$

Zeng, Xiao-ming, 0J, 11, 14, 17, 18

Zhai, Zhao-Hui, $4 \mathrm{O}$

Zhang, Bin, OU, 4Q

Zhang, Dandan, 25

Zhang, Da-yong, 3L

Zhang, Fan, OE

Zhang, Feizhou, 1M, 2Z, 36

Zhang, Jia-lei, 4A

Zhang, Jianzhu, 1M, 36, 3R

Zhang, Jun, OG

Zhang, Junwei, 16, 18

Zhang, Junyong, 1P, 32, 4M

Zhang, Kai, OQ, 2Y, 3B, 4S

Zhang, Kuo, 2T, 3P

Zhang, Linwen, 4G

Zhang, Lisong, 4L

Zhang, Peng, 2J

Zhang, Pu, OW

Zhang, R., 05

Zhang, Rongzhu, $1 \mathrm{~L}$

Zhang, Rui, 4B

Zhang, Shaoqian, $2 \mathrm{M}$

Zhang, Wei, $\mathrm{OH}, 46$

Zhang, Wei, 1Q, 24

Zhang, X. M., 05

Zhang, Xin, 03

Zhang, Xin, 3E, 3I, 3J

Zhang, Xiongjun, OG

Zhang, Xuejie, 32

Zhang, Yanli, 1P, 32, 4M

Zhang, Yao, 44

Zhang, Ying, IF, $1 G$

Zhang, Yongliang, ON, 19

Zhang, Yongsheng, 23

Zhang, Yuelong, 26, 2J, 2P

Zhang, Zeng-Bao, 1U, 2Q, 3Y

Zhang, Zhi-Guo, 1U, 2Q

Zhao, Jun, $2 X$

Zhao, Jun-pu, 3l

Zhao, Lei, OJ, ON, 11, 13, 19

Zhao, Liu, $2 X$

Zhao, Weili, 2J

Zhao, Xueqing, 23

Zhao, Zhiwei, 1D

Zheng, Changbin, 2T

Zheng, Jiangang, $0 G$

Zheng, Kuixing, $0 G$

Zheng, Wanguo, 3V

Zhong, Sen-Cheng, 40 
Zhong, Wei, OE

Zhong, Zheqiang, 4Q

Zhou, Canhua, 29, 2G, 2

Zhou, D. D., 05

Zhou, Dongjian, 29, 2G, 21, 30

Zhou, Hailiang, 4L

Zhou, Hang, $1 \mathrm{C}$

Zhou, Kai-nan, 0J, 11, 14, 16, 17, 18

Zhou, Li-dan, $1 R$

Zhou, Pu, OB

Zhou, Qiong, 35

Zhou, Rui, 38

Zhou, Shouhuan, 0C, 3W

Zhou, Song, 0J, 11, 14, 18

Zhou, Songqing, $2 X$

Zhou, Taidou, ON, 19

Zhou, Tangjian, $\mathrm{OL}$

Zhou, Wei, OE, 3E, 3I, 3J, 4B

Zhou, Wenchao, 3D

Zhou, Xinda, 3V

Zhou, Xuanfeng, 1C

Zhu, Feng, 22, 2X

Zhu, Jianqiang, 1P, 32, 34, 4M

Zhu, Li-Guo, 40

Zhu, N., 05

Zhu, Qihua, 0J, 11, 13, 16, 17, 18, 19, 3E, 4B, 4L

Zhu, Xiaolei, 08, OA, OD

Zhu, Yongxiang, 23

Zhu, Zhuoya, 1D

Zhuang, Xinyu, 3G

Zorn, M., OY

Zuo, Yan-lei, 0J, 11, 12, 14, 16, 17 


\title{
Conference Committee
}

\author{
Conference Chairs
}

Cangli Liu, China Academy of Engineering Physics (China)

Willy L. Bohn, BohnLaser Consult (Germany)

Robert Walter, Schafer Corporation (United States)

Jarmila Kodymova, Institute of Physics AS (Czech Republic)

Symposium Honorary Chair

Xiangwan Du, China Academy of Engineering (China)

International Scientific Committee

Cangli Liu (China)

Kerim R. Allahverdi (Turkey)

Petar Atanasov (Bulgaria)

Willy L. Bohn (Germany)

Anatoly Boreysho (Russian Federation)

Jarmila Kodymova (Czech Republic)

Robert Walter (United States)

Dieter Schuöcker (Austria)

International Advisory Committee

Richard Ackerman (United States)
Kerim R. Allahverdi (Turkey)
Victor Apollonov(Russian
Federation)
Petar Atanasov (Bulgaria)
Michel Autric (France)
Boris Barmashenko (Israel)
Willy L. Bohn (Germany)
Anatoly Boreysho (Russian
Federation)
David Carroll (United States)
Hans Eichler (Germany)
Massamori Endo (Japan)
Andrey lonin (Russian Federation)
Koichi Kasuya (Japan)
Jarmila Kodymova (Czech
Republic)
Paolo Di Lazzaro (Italy)
Antonio Lapucci (Italy)

\author{
Bincheng Li (China) \\ Kazuo Maeno (Japan) \\ Mohammad Hossein Mahdieh (Iran, \\ Islamic Republic of) \\ Richard P. Mildren (Australia) \\ Vladislav Panchenko (Russian \\ Federation) \\ Zamik Rosenwaks (Israel) \\ Nikola Sabotinov (Bulgaria) \\ Fengting Sang (China) \\ Dieter Schuöcker (Austria) \\ Chun Tang (China) \\ Greg Tallents (United Kingdom) \\ Victor Tarasenko (Russian \\ Federation) \\ Keith Truesdell (United States) \\ Robert Walter (United States) \\ Kazuhiro Watanabe (Japan)
}


Local Organizing Committee

Guobin Fan, China Academy of Engineering Physics (China)

Zhixin Tan, China Academy of Engineering Physics (China)

Wanguo Zheng, Research Center of Laser Fusion, CAEP (China)

Yuqi Jin, Dalian Institute of Chemical Physics, CAS (China)

Hu Yang, Institute of Optics and Electronics, CAS (China)

Weibiao Chen, Shanghai Institute of Optics and Fine Mechanics, CAS

(China)

Mali Gong, Tsinghua University (China)

Guoying Feng, Sichuan University (China)

Jianheng Zhao, Institute of Fluid Physics, CAEP (China)

Xiaojian Shu, Beijing Institute of Applied Physics and Computational Mathematics (China)

Kai Zhang, Institute of Applied Electronics, CAEP (China)

\section{Session Chairs}

1 Solid State Laser Sources and System Engineering (Including Disk,

Fiber, Hybrid Lasers)

Chun Tang (China)

Leon Glebov (United States)

Mali Gong (China)

2 High Power Diode Laser

Richard P. Mildren (Australia)

3 Ultra-Short Pulsed Laser Systems, Theory, and Simulation (UV, VUV, EUV Lasers

Mohammad Hossein Mahdieh (Iran, Islamic Republic of)

Oliver de Vries (Germany)

$4 \quad$ Gas and Chemical Lasers (Including DPALs)

Boris D. Barmashenko (Israel)

Yuqi Jin (China)

5 Resonators and Laser Beam Control

Bincheng Li (China)

Timothy Madden (United States)

6 Applications, Laser Materials (Crystal, Ceramic), Novel Approaches

Guoying Feng (China)

Claude R. Phipps (United States) 


\section{Introduction}

The 20th International Symposium on High Power Systems \& Applications 2014 (HPLS\&A 2014) was held 25-29 August 2014, in Chengdu, China. It is the first time that the HPLS\&A symposium was held outside of Europe in Asia. The oral sessions and poster sessions covered the fields of solid state laser sources and system engineering (including disk, fiber, hybrid lasers); high power diode laser, gas and chemical lasers (including DPALs); resonators and laser beam control; ultra-short pulsed laser system, theory and simulation; laser materials (crystal, ceramic), UV, VUV, EUV lasers; as well as applications and novel approaches.

The symposium provided an excellent opportunity for researchers in gas, chemical, and high power lasers, along with the end-users of these lasers to exchange ideas, review the state of the art, and foresee emerging trends within the fields. Overall, the sessions were well attended and the presentations were followed by interesting and insightful discussions.

These proceedings, which provide a detailed account of the symposium, will be useful for the scientists, researchers, and engineers in the fields of high power laser systems and applications.

\section{Cangli Lliu \\ Willy L. Bohn \\ Robert Walter Jarmila Kodymova}


Proc. of SPIE Vol. 9255 925501-22

Downloaded From: https://www.spiedigitallibrary.org/conference-proceedings-of-spie on 25 Apr 2023 Terms of Use: https://www.spiedigitallibrary.org/terms-of-use 